\title{
A system for the automatic estimation of morphometric parameters of corneal endothelium in alizarine red stained images
}

\author{
Alfredo Ruggeri", Fabio Scarpa ${ }^{\#}$, Massimo De Luca ${ }^{\#}$, \\ Christian Meltendorf ${ }^{\wedge}$, Jan Schroeter* \\ \# Department of Information Engineering, University of Padua, \\ Padua, Italy \\ `Department of Ophthalmology, Martin-Luther-University, Halle, Germany \\ *Department of Ophthalmology, Charité-Universitätsmedizin Berlin, Campus Virchow- \\ Klinikum, Berlin, Germany
}

Corresponding author: Prof. Alfredo Ruggeri, Department of Information Engineering, University of Padua, Via Gradenigo 6/B, 35131 Padua, Italy. Tel: +39 049827 7624, Fax: +39 049827 7699, E-mail: alfredo.ruggeri@unipd.it

Keywords: morphometry, cornea endothelium, alizarine red, image analysis.

Word count (excluding title page, abstract, tables and figures, acknowledgements and references): 2421. 


\begin{abstract}
Background/aims. A computer program for the automatic estimation of morphometric parameters (cell density, pleomorphism, polymegethism) in alizarine red stained images is presented and evaluated.

Methods. Images of corneal endothelium from 30 porcine eyes stained with alizarine red were acquired with an optical microscope and saved as grey-level digital images. Each image was at first pre-processed for luminosity correction and contrast enhancement. An artificial neural network was used to classify all pixels as cell contour or cell body pixels. The segmented cell contours were then used to obtain estimates of morphometric parameters. The central area was assessed and the mean area per cornea was $0.54 \pm 0.07 \mathrm{~mm}^{2}$. The whole system was implemented as a computer program using the Matlab ${ }^{\circledR}$ language. Estimated parameters were compared with the corresponding values derived from manual contour detection on the same images used for the automatic estimation.

Results. On the 30 images of our data set, mean differences of automatic parameters vs. manual ones were $-12 \pm 52$ cells $/ \mathrm{mm}^{2}$ (range -103 to +145 ) for density; $0.5 \pm 2.6$ per cent (range -5.6 to +5.6 ) for pleomorphism; $-0.7 \pm 1.9$ per cent (range -4.1 to +2.8 ) for polymegethism.

Conclusion. The evaluation of the automatic system on 30 images from porcine eyes confirmed its capability of reliably estimating morphometric parameters with respect to parameter values derived by manual analysis.
\end{abstract}




\section{INTRODUCTION}

The evaluation of the main morphometric parameters of corneal endothelium provides information to assess the cornea health state. Cell density (ECD), polymegethism, i.e., differences in cell size often expressed as coefficient of variation (or fractional standard deviation) of cell areas, and pleomorphism (or hexagonality coefficient), defined as the fraction of hexagonal cells over the total number of cells, allow the quantitative characterization of the endothelial cell conditions. All these parameters require the reliable identification of cell contours in endothelial images. Computer programs have been proposed to accomplish this task since almost 30 years ago, see e.g. [1-5], even if at the best of our knowledge all such programs only provide a semi-automatic analysis.

In order to increase cell contour visibility, and thus to allow a more reliable estimation of the morphometric parameters above, dyes such as alizarine red are used in many in-vitro studies. In one such study, the quality control in an eye bank setting was carried out to validate the entire process from cornea procurement up to transplantation [6]. An uncontrolled change of temperature, which may e.g. occur during transportation, can actually exert a potentially harmful non-physiological influence on the corneal endothelial cells [7]. In order to study the effects of significant changes of the storage temperature in organ cultured corneas, an investigation has been performed, where alizarine red stained images were acquired and analyzed by corneal experts, who manually outlined, i.e. segmented, the cell contours to derive an estimation of the morphometric parameters [6].

Although in stained images cell contours are more visible and better outlined, their manual tracing, albeit performed in a computer-assisted manner, still remains a very long and tedious task. This often leads the experimenter to reduce the number of outlined cells to just a few tens, 
greatly affecting the accuracy of estimated parameters and thus the reliability of the study outcomes.

To overcome this situation, we have developed and evaluated a totally automatic computerized system that allows the reliable estimation of endothelium morphometric parameters in alizarine red stained images.

\section{MATERIAL AND METHODS}

\section{Image acquisition}

The corneas were obtained from commercially slaughtered pigs. The corneo-scleral discs were prepared under sterile conditions after disinfections in the laminar airflow unit: $8 \mathrm{~mm}$ central corneal disc were prepared using a corneal punch device (Katena Products, Denville, NJ, USA) and then transferred with the endothelium turned upward to a $50 \mathrm{ml}$ polystyrene container (Gosselin Plastiques, Hazebrouck Cedex, France) with $20 \mathrm{ml}$ dextran-containing culture medium (100 ml MEM with Earle's salts (10x); $20 \mathrm{ml}$ fetal bovine serum; $10 \mathrm{ml}$ penicillin/streptomycin $10.000 \mathrm{U} / 10.000 \mu \mathrm{g} / \mathrm{ml} ; 10 \mathrm{ml}$ amphotericin B $250 \mu \mathrm{g} / \mathrm{ml} ; 10 \mathrm{ml}$ L-glutamine (200 mM); 12.5 ml HEPES buffer $(1 \mathrm{M}) ; 29.3 \mathrm{ml}$ sodium bicarbonate (NaHCO3) (7.5\%); 60 g Dextran 500, $748.2 \mathrm{ml}$ aqua ad injectionem). The container was then closed air-tight and stored at $32^{\circ} \mathrm{C}$ for 24 hours.

Endothelial cell borders were visualized by staining with $0.2 \%$ alizarin red (Waldeck/Chroma, Münster, Germany) for 4 minutes. The $\mathrm{pH}$ of alizarin red was adjusted to 4.2 by using $0.1 \%$ ammonia solution [8]. The dye was then rinsed with $0.9 \%$ sodium chloride solution. Previous removal of the scleral ring enabled us to place the corneal discs on the microscope slides with the endothelium pointing downwards, and thus to display the 
endothelium on one level.

Endothelium images were obtained from 30 of such pig corneas using a calibrated inverse phase contrast microscope (CK 40, Olympus, Hamburg, Germany) at 200x magnification and an analog camera (SSC-DC50AP, Sony, Japan), and digitized as 768 × 576 pixels 8 bits gray-level images using the NAVIS software (Nidek Technologies, Padua, Italy). The calibration of the system was done using a standardized microscale (Neubauer chamber). The mean area assessed per cornea was $0.54 \pm 0.07 \mathrm{~mm}^{2}$ (range 0.31 to $0.64 \mathrm{~mm}^{2}$ ) and was located in the central zone of the cornea, to avoid bias in the cell morphometric evaluation [9]. In Fig. 1, top panel, a representative image (nr. 7) from our dataset is shown.

All 30 images of this dataset are publicly available for download [10].

\section{Automatic estimation of morphometric parameters}

A specific algorithm to identify cell contours was developed and implemented as a computer program. The flow diagram of Fig. 2 illustrates the main steps of the image analysis algorithm.

Image pre-processing. In order to exclude the peripheral region of the images, where contrast is lower and often prevents a reliable detection of cell contours, and to keep the number of analyzed cells reasonably low, a region of interest (ROI) was manually chosen in each image, located approximately in the center of the image, and used for both automatic and manual analysis (see below). In each image, this ROI includes on average 305 cells (range 223 to 442 cells), in order to allow a reliable estimate of the morphometric parameters, and covers an average area of $0.101 \mathrm{~mm}^{2}$.

The images from corneal microscopes usually present a non-uniform illumination and some pixel noise. In order to cope with these problems, a parabolic correction was at first used to 
improve luminosity equalization and contrast enhancement. The compensation of the illumination drift was obtained by fitting it with a parabolic function, which is then subtracted from the image, both horizontally and vertically. A band-pass filtering (with cutoff frequencies of $0.02 f_{c}$ and $0.2 f_{c}$, where $f_{c}$ is the spatial sampling frequency of the image) was then applied to enhance the space frequency components specifically related to cell contour detection and to reduce the ones related to noise. Contrast, especially the one between dark cell boundaries and light cell bodies, is generally very low in this type of images. Local contrast was further enhanced by applying to each image pixel a sigmoid point transformation, centered on the image mean gray-level value.

Cell contour segmentation. A multi-layer feed-forward artificial neural network (ANN), with local shift invariant interconnections [11], was used to obtain a binary classification of all image pixels as either cell contour or cell body pixels. Each single pixel of the image was considered and its 121 adjacent pixels (11x11 pixels square neighborhood) were used to extract 7 features, used to classify the pixel as belonging to one of 8 possible classes: cell body; vertical border; horizontal border; oblique border; border with angle towards left; border with angle towards right; border with angle up; border with angle down. Finally, each pixel was classified into cell body (if assigned to the first class) or cell boundaries (if assigned to any of the other 7 classes). The adopted network is composed of 1 input, 2 hidden, and 1 output layers, with 7, 7, and 1 nodes respectively.

The ANN was trained on a set of 5 samples, randomly selected from different images of the dataset; each sample is made of a pair of 120x120 pixels sub-images: the original sub-image, cropped from the acquired image, and the same sub-image with the cell contour manually outlined by a cornea imaging expert. 
Cell contour post-processing. The resulting ANN, trained as described above, was applied to the images to obtain a first segmentation of cell contours. This segmented image was then skeletonised [12] to accurately extract the cell contours as 1-pixel thick lines. Unfortunately, grey-level information alone is often not sufficient to correctly detect cell contours. To cope with the errors still present in the segmented image (missing contours or false contours), a postprocessing procedure was developed based on an automatic, multi-step approach, which includes missing contour recovery and tentative merging and splitting of cell bodies. For every identified cell, a set of features was evaluated, such as mean radius, standard deviation of radius, standard deviation of relative radius (radius / mean radius), aspect ratio (ratio of the two main diagonals). Trying to improve an overall 'cell score', based on the values of these features, attempts were made to fuse small adjacent cells or to split large cells. This was done according to the general paradigm of trying to obtain cells whose shape is close to that of regular polygons: any operation (splitting or fusion) that improves this regularity is accepted. No manual correction of any type was applied to the obtained cell contours.

Computation of morphometric parameters. From the contour images eventually obtained, area and number of sides of all detected cells were straightforwardly derived. From these values, the estimation of ECD, pleomorphism, and polymegethism was finally carried out. ECD was computed as the sum of individual cell areas divided by the total number of cells; pleomorphism was computed by counting for each cell the number of neighboring cells (cells on the border of the considered ROI were excluded from this computation) and taking the percentage of cells with hexagonal shape; polymegethism was computed as the fractional standard deviation of all cell areas.

The whole system was developed as a collection of computer program modules, implemented 
using the Matlab ${ }^{\circledR}$ computer language (Matlab v. R2008, http://www.mathworks.com/).

\section{Manual estimation of morphometric parameters}

In order to assess the accuracy of the morphometric parameters estimated by the computerized procedure described above, ground truth reference values were obtained on the same ROIs with a manual estimation of the parameters performed by a cornea imaging expert. Using a public-domain image manipulation program (GIMP v. 2.6, http://www.gimp.org/) on screen-displayed enlarged version of the images, all visible cell contours were drawn, so as to outline the polygonal shape of each cell. The same procedure used in the automatically segmented images (see above) was then used to estimate the morphometric parameters also in the manually segmented ones.

All the manually segmented images of this dataset are publicly available for download [10].

\section{RESULTS}

Table 1 reports the statistics for the three morphometric parameters using the manual or the automatic method and the differences between the two methods. As regards ECD, these differences have an average value of 12 cells $/ \mathrm{mm}^{2}$, with a range from -103 cells $/ \mathrm{mm}^{2}$ to +145 cells $/ \mathrm{mm}^{2}$, respectively. For pleomorphism these values are 0.5 per cent, -5.6 per cent and +5.6 per cent, respectively; for polymegethism they are -0.7 per cent, -4.1 per cent and +2.8 per cent, respectively. The same statistics for the percent differences (with respect to manual values, assumed as reference values) are: $0 \%,-1 \%$ and $+3 \%$ for $\mathrm{ECD} ; 1 \%,-11 \%$, and $+13 \%$ for pleomorphism; $-3 \%,-17 \%$, and $+13 \%$ for polymegethism, respectively.

Figs. 2 shows the scatter-plots of manually vs. automatically estimated parameters. From this graphical representation of the results, the correlation between the automatic and manual method 
can be appreciated and quantitatively described by the Pearson's correlation coefficient, with values of 0.99 for ECD and 0.80 for both pleomorphism and polymegethism. To display the extent of agreement between the two methods, Bland-Altman plots [13, 14] of differences vs. averages for each pair of manual and automatic parameters are shown in Fig. 3, where lines indicate the average difference and the $95 \%$ limits of agreement: $11.9,-90.4$ and 114.3 cells $/ \mathrm{mm}^{2}$ for ECD; $0.48,-4.54$ and +5.50 per cent for pleomorphism; $-0.69,-4.33,+2.95$ per cent for polymegethism, respectively.

One representative example of the contours obtained by the proposed algorithm is shown in Fig. 1, bottom panel. The average run time of the current Matlab ${ }^{\circledR}$ prototype on a single image is 3 minutes.

\section{DISCUSSION AND CONCLUSION}

We have presented here a system for the estimation of cornea endothelium morphometric parameters that is completely automated and requires no manual editing by the user. The estimates of the parameters provided by the proposed algorithm are in very good agreement with the reference ones, obtained with careful manual analysis.

The estimation of ECD can nowadays be performed with reasonably good accuracy with some computerized analysis systems, see e.g. $[15,16]$, as the possible presence of a few errors in cells/contours detection is of limited impact on the final ECD estimation. On the contrary, as already noted by other authors, e.g. [17], the quantitative estimation of pleomorphism and polymegethism is much more affected than ECD by the wrong contour detection even in very few cells, rendering the reliable estimation of these parameters quite difficult. A possible solution is the manual correction of cell borders, but this might require a significant amount of 
work, e.g., on about 50 to 75 per cent of cell borders [17]. For the system we propose, the $95 \%$ limits of agreement for pleomorphism and polymegethism estimation were always below 5 per cent (in actual calculated value), making the results automatically provided by the system reliable enough to reach clinically significant conclusions. It must be noted, however, that at variance with other proposed systems, contour recognition in this in-vitro study was easier than in in-vivo clinical situations, where a dye cannot be used to increase cell visibility. For this reason, the present version of our system can be used only in experimental settings and not in cornea banking or clinical applications on patients.

In large experimental studies employing alizarine red dye, such as the one addressed here, hundreds of images may need to be analyzed. In this case, for the practical reasons mentioned above, the experimenter usually restricts him/herself to estimate only ECD. The system we propose may thus be extremely valuable, as it can provide the experimenter with reliable estimation of the other two important morphometric parameters. Moreover, with respect to routine manual or semi-automatic analysis, where usually just a few tens of contiguous cells are considered, the proposed algorithm can process images containing hundreds of contiguous cells, to provide higher accuracy for the estimated morphometric parameters. This latter can be further increased by selecting in the central part of the cornea many images, positioned at slightly different locations, each one with hundreds of cells.

The present version of the proposed system, still a research prototype, would require a sizable amount of time to perform such a multi-image analysis. Implementation of the whole system in the more efficient computer language $\mathrm{C}++$ would reduce the execution time to the order of 10 seconds per image, allowing multi-image analysis to be performed within one minute. In addition, in this latter version a user-friendly editing interface might be included, to 
allow the user to quickly manually correct the possible residual errors in contour detection (on average, in 1 per cent of cells only) and reach a 100\% accuracy in parameter estimation (with respect to manual analysis).

Albeit the in-vitro studies addressed here are very important to evaluate and compare different experimental situations, they are however very specific and performed less often than clinical applications. In order to provide an automatic evaluation of morphometric parameters also in clinical images, we are developing a version of our system able to analyze endothelium images acquired in patients, e.g. with specular or confocal microscopes.

\section{Competing Interest: None declared.}

\section{Licence for Publication}

The Corresponding Author has the right to grant on behalf of all authors and does grant on behalf of all authors, an exclusive licence (or non exclusive for government employees) on a worldwide basis to the BMJ Publishing Group Ltd to permit this article (if accepted) to be published in BJO and any other BMJPGL products and sublicences such use and exploit all subsidiary rights, as set out in our licence (http://group.bmj.com/products/journals/instructions-for-authors/licenceforms). 


\section{REFERENCES}

[1] Lester JM, McFarland JL, Bursell SE, et al. Automated morphometric analysis of corneal endothelial cells. Invest Ophthalmol Vis Sci 1981; 20:407-10.

[2] Koyama T, Matsuo N, Sakaguchi N, Asano H, Yuasa K, Tsuchida Y. Image analysis of morphologic features of the corneal endothelium including hexagonality. Nippon Ganka Gakkai Zasshi. 1990; 94(10):951-6.

[3] Oblak E, Doughty MJ, Oblak L. A semi-automated assessment of cell size and shape in monolayers, with optimal adjustment for the cell-cell border width - application to human corneal endothelium. Tissue Cell 2002; 34(4):283-295.

[4] Giasson CJ. Graham A, Blouin J, Solomon L, Gresset J, Melillo M, Polse KA. Morphometry of cells and guttae in subjects with normal or guttate endothelium with a contour detection algorithm. Eye Contact Lens 2005; 31(4):158-165.

[5] Siertsema JV, Landesz M, van den Brom H, van Rij G. Automated video image morphometry of the corneal endothelium. Doc Ophthalmol 1993; 85(1):35-44.

[6] Schroeter J, Meltendorf C, Ohrloff C, Rieck P. Influence of temporary hypothermia on corneal endothelial cell density during organ culture preservation. Graefes Arch Clin Exp Ophthalmol 2008; 264(3):369-72.

[7] Madden PW, Maguire SH. Methods of refrigerated transport of corneas for transplantation. Aust NZ J Ophthalmol 1996; 24:17-20.

[8] Taylor MJ, Hunt CJ. Dual staining of corneal endothelium with trypan blue and alizarin red S: importance of $\mathrm{pH}$ for the dye-lake reaction. Br J Ophthalmol 1981; 65:815-819.

[9] Amann J, Holley GP, Lee S, et al. Increased endothelial cell density in the paracentral and peripheral regions of the human cornea. Am J Ophthalmol 2003; 135:584-590.

[10] http://bioimlab.dei.unipd.it/Data Sets.htm

[11] Long X, Cleveland WL, Yai YL. Effective automatic recognition of cultured cells in bright field images using Fisher's linear discriminant preprocessing. Images and Video Computing 2005; 23:1203-1213.

[12] Gonzalez RC, Woods RE. Digital Image Processing. Prentice Hall, 2002.

[13] Bland JM, Altman DG. Statistical methods for assessing agreement between two methods of clinical measurement. Lancet 1986; 1:307-310.

[14] Bland JM, Altman DG. Measuring agreement in method comparison studies. Stat Methods Med Res 1999; 8:135-160.

[15] Ruggeri A, Grisan E, Jaroszewski J. A new system for the automatic estimation of endothelial cell density in donor corneas. Br J Ophthalmol 2005; 89:306-311.

[16] Gain P, Thuret G, Gavet Y, et al. Automated tri-image analyser of stored corneal endothelium. Br J Ophthalmol 2002; 86: 801-808.

[17] Doughty MJ, Aakre BM. Further analysis of assessments of the coefficient of variation of corneal endothelial cell areas from specular microscopic images. Clin Exp Optom 2008; 
91:438-446. 
Table and figure legends

Table 1. Summary results of automatic parameter estimation. For each cornea, manual and automatic values are reported for all three parameters, as well as their difference ('Diff'), with percent values ('\%’), and absolute difference ('Abs'), also with percent values ('\%”). For all quantities, average, standard deviation, minimum and maximum values are reported for the 30 cornea images. Cell densities values are in cells $/ \mathrm{mm}^{2}$, pleomorphism and polymegethism are per cent values.

Fig. 1. Original (top panel) and processed (bottom panel) image of alizarine red stained corneal endothelium (nr. 7). The black outline in the bottom panel image represents the cell contour generated by the proposed computer algorithm.

Fig. 2. Flow diagram of the main steps of the image analysis algorithm.

Fig. 3. Scatter-plot of automatic vs. manual estimates of ECD (left), pleomorphism (center) and polymegethism (right). The dashed line indicates the line of identity, the solid one the linear regression line.

Fig. 4. Scatter-plots of difference vs. average for each pair of manual and automatic ECD (left), pleomorphism (center) and polymegethism (right). The dotted line shows the average difference, the dashed lines show the $95 \%$ limits of agreement [12-13]. 


\begin{tabular}{|c|c|c|c|c|c|c|c|c|c|c|c|c|c|c|c|c|c|c|}
\hline Image & $\begin{array}{c}\text { Manual } \\
\text { ECD }\end{array}$ & $\begin{array}{c}\text { Automatic } \\
\text { ECD }\end{array}$ & Diff & $\%$ & Abs & $\%$ & \begin{tabular}{|c|} 
Manual \\
Pleomorphism \\
\end{tabular} & \begin{tabular}{|c|} 
Automatic \\
Pleomorphism \\
\end{tabular} & Diff & $\%$ & Abs & $\%$ & \begin{tabular}{|c|} 
Manual \\
Polymegethism \\
\end{tabular} & \begin{tabular}{|c|} 
Automatic \\
Polymegethism \\
\end{tabular} & Diff & $\%$ & Abs & $\%$ \\
\hline 1 & 4661 & 4658 & -3 & $0 \%$ & 3 & $0 \%$ & 44.5 & 45.5 & 1.0 & $2 \%$ & 1.0 & $2 \%$ & 25.9 & 24.0 & -1.9 & $-7 \%$ & 1.9 & $7 \%$ \\
\hline 2 & 4524 & 4643 & 119 & $3 \%$ & 119 & $3 \%$ & 49.8 & 52.8 & 3.0 & $6 \%$ & 3.0 & $6 \%$ & 27.0 & 25.0 & -2.0 & $-7 \%$ & 2.0 & $7 \%$ \\
\hline 3 & 4676 & 4701 & 25 & $1 \%$ & 25 & $1 \%$ & 46.8 & 46.6 & -0.2 & $0 \%$ & 0.2 & $0 \%$ & 24.7 & 27.1 & \begin{tabular}{|l|}
2.4 \\
\end{tabular} & $10 \%$ & 2.4 & $10 \%$ \\
\hline 4 & 3892 & 3895 & 3 & $0 \%$ & 3 & $0 \%$ & 52.3 & 49.6 & -2.7 & $-5 \%$ & 2.7 & $5 \%$ & 18.9 & 18.0 & -0.9 & $-5 \%$ & 0.9 & $5 \%$ \\
\hline 5 & 4405 & 4383 & -22 & $0 \%$ & 22 & $0 \%$ & 46.2 & 47.1 & 0.9 & $2 \%$ & 0.9 & $2 \%$ & 19.5 & 19.5 & 0.0 & $0 \%$ & 0.0 & $0 \%$ \\
\hline 6 & 4131 & 4062 & -69 & $-2 \%$ & 69 & $2 \%$ & 49.2 & 47.4 & -1.8 & $-4 \%$ & 1.8 & $4 \%$ & 19.0 & 16.6 & -2.4 & $-13 \%$ & 2.4 & $13 \%$ \\
\hline 7 & 4230 & 4188 & -42 & $-1 \%$ & 42 & $1 \%$ & 48.8 & 51.8 & 3.0 & $6 \%$ & 3.0 & $6 \%$ & 17.7 & 17.1 & -0.6 & $-3 \%$ & 0.6 & $3 \%$ \\
\hline 8 & 4419 & 4397 & -22 & $0 \%$ & 22 & $0 \%$ & 53.6 & 52.3 & -1.3 & $-2 \%$ & 1.3 & $2 \%$ & 19.0 & 19.1 & 0.1 & $1 \%$ & 0.1 & $1 \%$ \\
\hline 9 & 4895 & 4860 & -35 & $-1 \%$ & 35 & $1 \%$ & 48.1 & 51.6 & 3.5 & $7 \%$ & 3.5 & $7 \%$ & 20.3 & 20.3 & 0.0 & $0 \%$ & 0.0 & $0 \%$ \\
\hline 10 & 4587 & 4592 & 5 & $0 \%$ & 5 & $0 \%$ & 49.1 & 50.9 & 1.8 & $4 \%$ & 1.8 & $4 \%$ & 21.6 & 21.3 & -0.3 & $-1 \%$ & 0.3 & $1 \%$ \\
\hline 11 & 4171 & 4316 & 145 & $3 \%$ & 145 & $3 \%$ & 47.6 & 45.6 & -2.0 & $-4 \%$ & 2.0 & $4 \%$ & 23.5 & 24.8 & 1.3 & $6 \%$ & 1.3 & $6 \%$ \\
\hline 12 & 3971 & 4008 & 37 & $1 \%$ & 37 & $1 \%$ & 54.1 & 58.3 & 4.2 & $8 \%$ & 4.2 & $8 \%$ & 22.2 & 25.0 & 2.8 & $13 \%$ & 2.8 & $13 \%$ \\
\hline 13 & 3874 & 3843 & -31 & $-1 \%$ & 31 & $1 \%$ & 43.7 & 49.3 & 5.6 & $13 \%$ & 5.6 & $13 \%$ & 24.5 & 26.4 & \begin{tabular}{|l|}
1.9 \\
\end{tabular} & $8 \%$ & 1.9 & $8 \%$ \\
\hline 14 & 3759 & 3710 & -49 & $-1 \%$ & 49 & $1 \%$ & 41.2 & 41.2 & 0.0 & $0 \%$ & 0.0 & $0 \%$ & 23.6 & 23.1 & -0.5 & $-2 \%$ & 0.5 & $2 \%$ \\
\hline 15 & 3765 & 3817 & 52 & $1 \%$ & 52 & $1 \%$ & 45.5 & 46.2 & 0.7 & $2 \%$ & 0.7 & $2 \%$ & 24.9 & 23.6 & $\mid-1.3$ & $-5 \%$ & 1.3 & $5 \%$ \\
\hline 16 & 3703 & 3724 & 21 & $1 \%$ & 21 & $1 \%$ & 51.5 & 51.8 & \begin{tabular}{|l|}
0.3 \\
\end{tabular} & $1 \%$ & 0.3 & $1 \%$ & 24.8 & 21.9 & -2.9 & \begin{tabular}{|l|}
$-12 \%$ \\
\end{tabular} & 2.9 & $12 \%$ \\
\hline 17 & 3750 & 3773 & 23 & $1 \%$ & 23 & $1 \%$ & 49.5 & 46.6 & -2.9 & $-6 \%$ & 2.9 & $6 \%$ & 24.4 & 24.5 & \begin{tabular}{|l|}
0.1 \\
\end{tabular} & $0 \%$ & 0.1 & $0 \%$ \\
\hline 18 & 3910 & 3807 & -103 & $-3 \%$ & \begin{tabular}{|l|}
103 \\
\end{tabular} & $3 \%$ & 51.2 & 52.4 & \begin{tabular}{|l|}
1.2 \\
\end{tabular} & $2 \%$ & 1.2 & $2 \%$ & 26.9 & 25.1 & -1.8 & $-7 \%$ & 1.8 & $7 \%$ \\
\hline 19 & 3793 & 3848 & 55 & $1 \%$ & 55 & $1 \%$ & 52.0 & 50.2 & -1.8 & $-3 \%$ & 1.8 & $3 \%$ & 21.4 & 24.1 & 2.7 & $13 \%$ & 2.7 & $13 \%$ \\
\hline 20 & 4273 & 4302 & 29 & $1 \%$ & 29 & $1 \%$ & 49.4 & 43.8 & -5.6 & $-11 \%$ & 5.6 & $11 \%$ & 25.5 & 26.3 & 0.8 & $3 \%$ & 0.8 & $3 \%$ \\
\hline 21 & 4310 & 4379 & 69 & $2 \%$ & 69 & $2 \%$ & 48.6 & 52.6 & 4.0 & $8 \%$ & 4.0 & $8 \%$ & 24.8 & 23.2 & -1.6 & $-6 \%$ & 1.6 & $6 \%$ \\
\hline 22 & 4426 & 4503 & 77 & $2 \%$ & 77 & $2 \%$ & 47.3 & 45.3 & -2.0 & $-4 \%$ & 2.0 & $4 \%$ & 25.0 & 24.8 & -0.2 & $-1 \%$ & 0.2 & $1 \%$ \\
\hline 23 & 3828 & 3804 & -24 & $-1 \%$ & 24 & $1 \%$ & 48.0 & 50.0 & 2.0 & $4 \%$ & 2.0 & $4 \%$ & 22.9 & 18.9 & -4.0 & $-17 \%$ & 4.0 & $17 \%$ \\
\hline 24 & 4057 & 4082 & 25 & $1 \%$ & 25 & $1 \%$ & 44.2 & 43.8 & -0.4 & $-1 \%$ & 0.4 & $1 \%$ & 23.3 & 21.5 & -1.8 & $-8 \%$ & 1.8 & $8 \%$ \\
\hline 25 & 4209 & 4226 & 17 & $0 \%$ & 17 & $0 \%$ & 48.5 & 49.3 & 0.8 & $2 \%$ & 0.8 & $2 \%$ & 27.6 & 28.5 & 0.9 & $3 \%$ & 0.9 & $3 \%$ \\
\hline 26 & 3960 & 3938 & -22 & $-1 \%$ & 22 & $1 \%$ & 42.5 & 41.5 & -1.0 & $-2 \%$ & 1.0 & $2 \%$ & 28.4 & 25.4 & -3.0 & $-11 \%$ & 3.0 & $11 \%$ \\
\hline 27 & 4283 & 4331 & 48 & $1 \%$ & 48 & $1 \%$ & 40.2 & 38.0 & -2.2 & $-5 \%$ & 2.2 & $5 \%$ & 24.9 & 20.8 & -4.1 & $-16 \%$ & 4.1 & $16 \%$ \\
\hline 28 & 4065 & 4092 & 27 & $1 \%$ & 27 & $1 \%$ & 46.9 & 50.4 & 3.5 & $7 \%$ & 3.5 & $7 \%$ & 22.2 & 21.9 & -0.3 & $-1 \%$ & 0.3 & $1 \%$ \\
\hline 29 & 3857 & 3869 & 12 & $0 \%$ & 12 & $0 \%$ & 48.1 & 47.9 & -0.2 & $0 \%$ & 0.2 & $0 \%$ & 26.3 & 23.2 & -3.1 & $-12 \%$ & 3.1 & $12 \%$ \\
\hline 30 & 4097 & 4088 & -9 & $0 \%$ & 9 & $0 \%$ & 49.5 & 52.5 & 3.0 & $6 \%$ & 3.0 & $6 \%$ & 23.6 & 22.6 & $\mid-1.0$ & $-4 \%$ & 1.0 & $4 \%$ \\
\hline average & 4149 & 4161 & 12 & \begin{tabular}{|l|}
$0 \%$ \\
\end{tabular} & 41 & $1 \%$ & 47.9 & 48.4 & 0.5 & $1 \%$ & 2.1 & $4 \%$ & 23.5 & 22.8 & \begin{tabular}{|l|} 
\\
\end{tabular} & $-3 \%$ & 1.6 & $7 \%$ \\
\hline sd & 320 & 330 & 52 & \begin{tabular}{|l|}
$1 \%$ \\
\end{tabular} & 34 & $1 \%$ & 3.4 & 4.3 & 2.6 & $5 \%$ & 1.5 & $3 \%$ & 2.8 & 3.0 & \begin{tabular}{|l|}
1.9 \\
\end{tabular} & $8 \%$ & 1.2 & $5 \%$ \\
\hline $\min$ & 3703 & 3710 & \begin{tabular}{|l|}
-103 \\
\end{tabular} & $-3 \%$ & 3 & $0 \%$ & 40.2 & 38.0 & -5.6 & \begin{tabular}{|l|}
$-11 \%$ \\
\end{tabular} & 0.0 & $0 \%$ & 17.7 & 16.6 & -4.1 & $-17 \%$ & 0.0 & $0 \%$ \\
\hline $\max$ & 4895 & 4860 & 145 & $3 \%$ & 145 & $3 \%$ & 54.1 & 58.3 & 5.6 & \begin{tabular}{|l|}
$13 \%$ \\
\end{tabular} & 5.6 & $13 \%$ & 28.4 & 28.5 & 2.8 & $13 \%$ & 4.1 & $17 \%$ \\
\hline
\end{tabular}



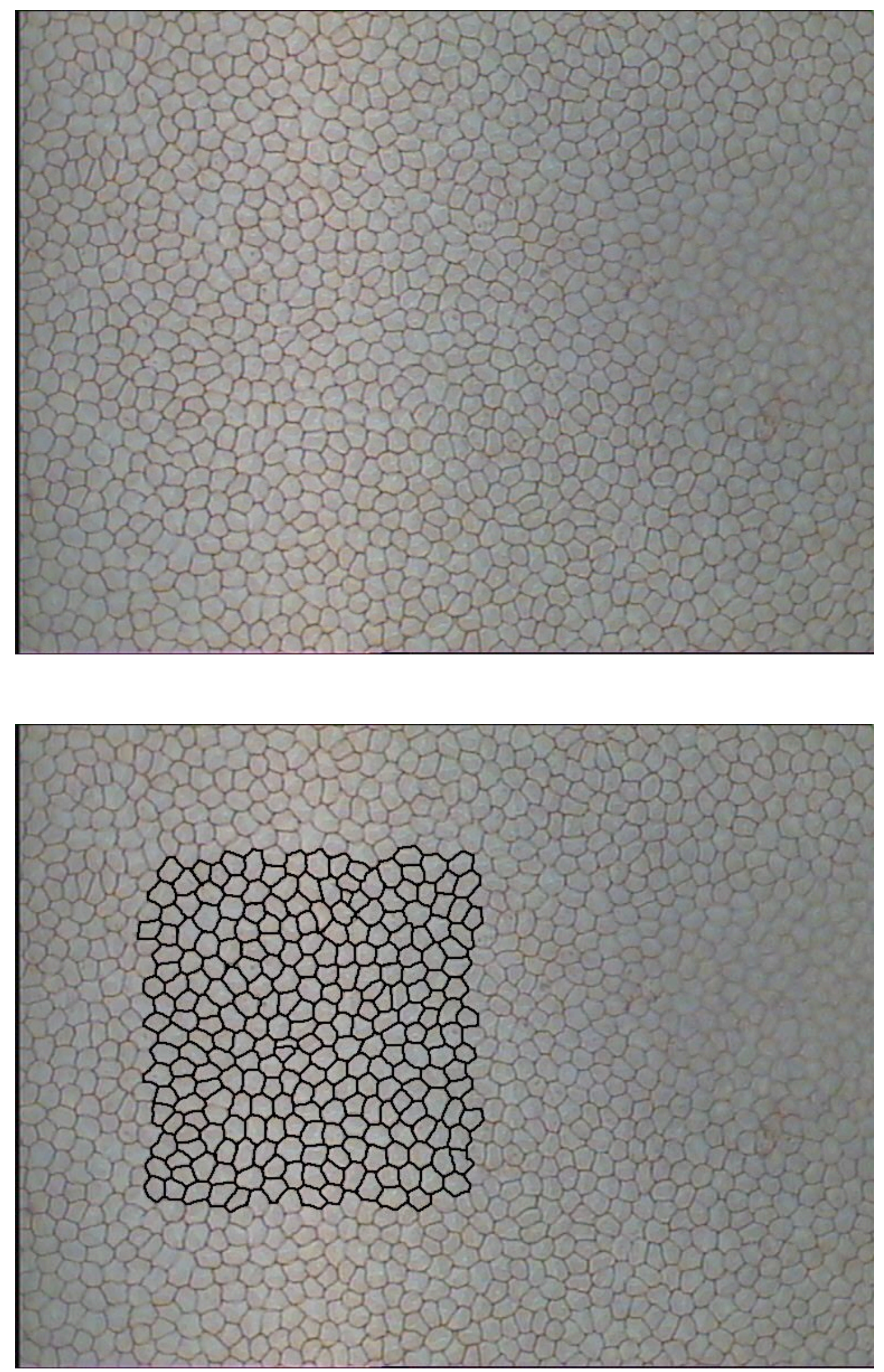
Luminosity equalization, band-pass filtering, sigmoid transformation

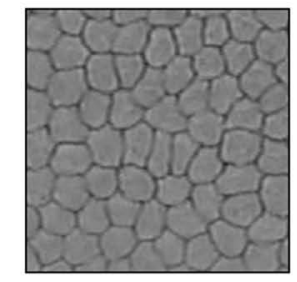

Artificial neural network

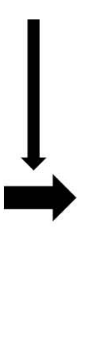

Post-processing procedure

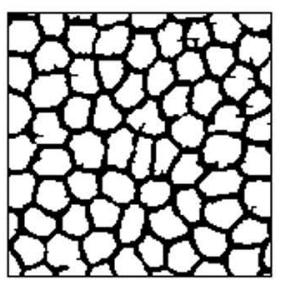

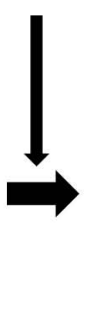

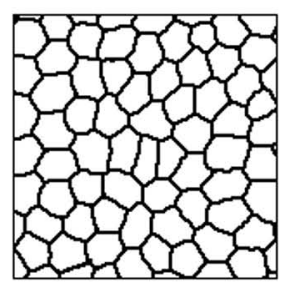



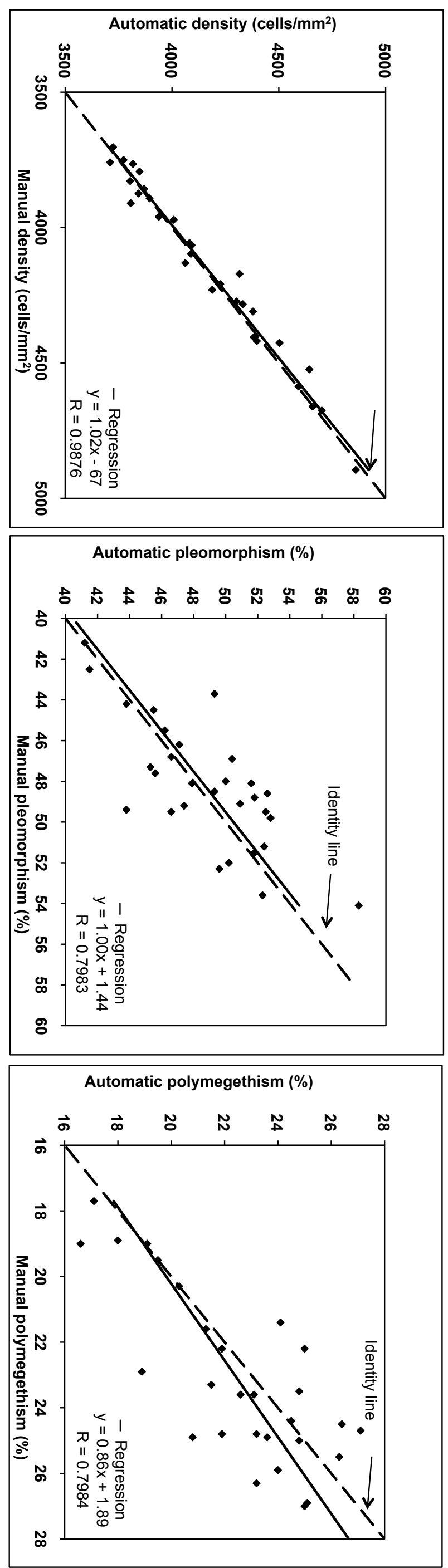

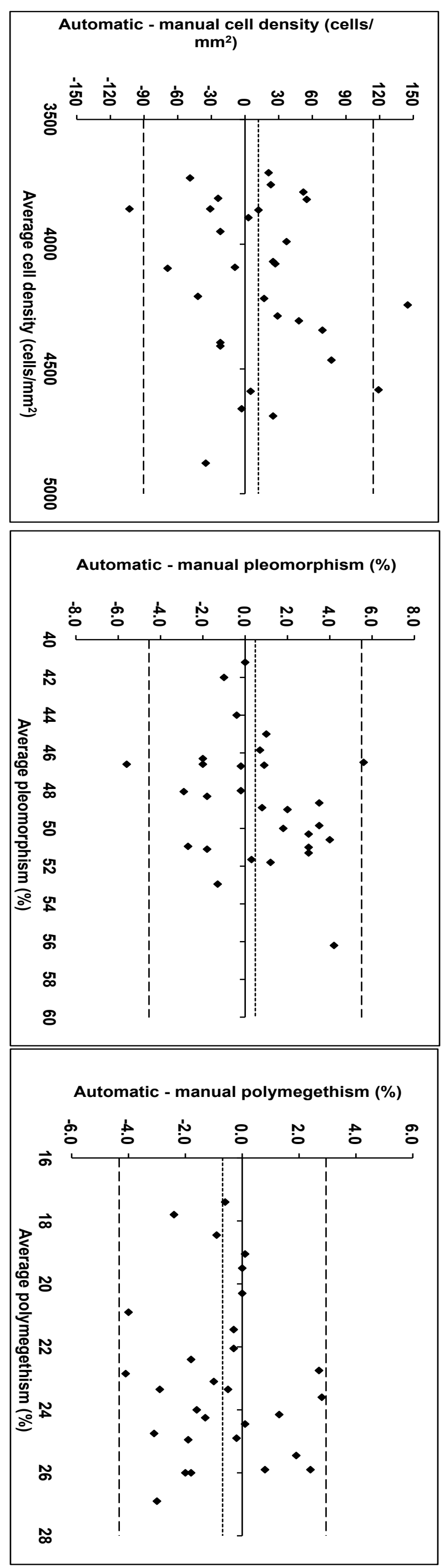